\title{
ON THE HOLOMORPHICAL CLASSIFICATION OF SPACES OF HOLOMORPHIC GERMS
}

\author{
JORGE ARAGONA
}

\section{Introduction}

Let $K$ be a compact set in a complex metrizable locally convex space $E$ and $F$ a complex Banach space. The study of the space of holomorphic germs $\mathscr{H}\left(K ; F^{\prime}\right)$ endowed with the Nachbin topology $\mathscr{T}_{\text {。 }}$ was undertaken by several authors. In particular by Chae [Ch] when $E$ is a Banach space; by Mujica $[\mathrm{Mu}]$ and Avilés [A-Mu] when $E$ is a metrizable locally convex space subject to some supplementary conditions and $F=C$; also by Soraggi [S], Wanderley [W] and more recently by Bierstedt and Meise [Bi-Me]. The point of view adopted in those works was, in a way, the "linear classification" of the spaces $\mathscr{H}(K ; F)$. In fact, in those papers results are obtained furnishing information about the behavior of the spaces $\mathscr{H}(K ; F)$ with respect to the following properties: bornological, barreled, complete, $(D F)$, Montel, Schwartz, Silva, nuclear, metrizable, etc. On the other hand, bounded and relatively compact subsets of the spaces $\mathscr{H}(K ; F)$ are studied and these results are applied to obtain properties of holomorphic mappings. This work is a contribution to the study of the "holomorphic classification" of the spaces $\mathscr{H}(K ; F)$, that is, we obtain results which give information about the behavior of these spaces with respect to the following properties: holomorphically bornological (hbo), holomorphically barreled (hba), holomorphically infrabarreled (hib) and holomorphically Mackey (hM), introduced in [N1], [N2] and [BMN].

We now describe briefly the content of this work.

In $\S 0$ we collect the basic definitions, notations and conventions of terminology that we shall use in the following sections.

In $\S 1$ we introduce the polynomially bornological (pbo), polynomially barreled (pba), polynomially infrabarreled (pib) and polynomially Mackey (pM) spaces. These properties are intermediate between the properties hbo,

Received December 14, 1979. 
hba, hib, hM and bornological, barreled, infrabarreled and Mackey, respectively. The study of the relations between these polynomial properties and the corresponding holomorphic properties leads up naturally to the concepts of $D$-holomorphy (and property $(D)$ ) and $D^{*}$-holomorphy (and property $\left(D^{*}\right)$ ). Interest in the polynomial properties is based on two facts. On the one hand, as we prove in $\S 4$, the spaces $\mathscr{H}(K ; F)$ have these properties. On the other hand-since we have got to reconstitute the holomorphical properties (hbo, hba, hib) starting from the corresponding polynomial properties and properties $(D)$ and $\left(D^{*}\right)$-we can express the properties "hbo", "hba" and "hib" as a union of more simple properties which, seemingly, should simplify the problem of the holomorphic classification of the spaces $\mathscr{H}(K ; F)$. This section ends with the introduction of holomorphically semibornological spaces.

In $\S 2$, after several results, we prove that a polynomially bornological space $E$ is holomorphically bornological if and only if $\left(\mathscr{H}(U ; F) ; \mathscr{T}_{0}\right)$ is complete for all equilibrated open subsets $U$ of $E$ and all complete Hausdorff locally convex spaces $F$. Combining these results with another one from $\S 1$, we get the principal results of this section (Theorem 2.6).

In $\S 3$ we prove that the Montel (resp. infra-Montel) property implies property $\left(D^{*}\right)$ (resp. $(D)$ ). From these results we deduce several relations between the Montel properties and the holomorphically significant properties, for spaces having polynomially significant properties.

In $\S \S 1,2$ and 3 the exposition is for arbitrary locally convex spaces.

It is only in $\S 4$ that we consider the problem of the holomorphic classification of the spaces $\mathscr{H}(K ; F)$. In this section, to avoid complicated notations on spaces of germs, we study, from the view point of holomorphic classification, the Cauchy regular inductive limits which is a class of locally convex spaces with the principal structural properties of the spaces of holomorphic germs usually studied. We prove that every Cauchy regular inductive limit is polynomially bornological and polynomially barreled. We prove also that, for a Cauchy regular inductive limit, properties $(D)$ and $\left(D^{*}\right)$ are equivalent. These results combined with several results of the preceding sections, lead to the main result of this paper (Theorem 4.6) which states that for a Cauchy regular inductive limit $E$ properties "hbo", "hba", "hib", $(D),\left(D^{*}\right)$, "Montel", "infra-Montel", "completeness of $\left(\mathscr{H}(U ; F) ; \mathscr{T}_{0}\right)$ for all non-void open subsets $U$ of $E$ and all complete Hausdorff locally convex spaces $F "$ and several others properties, are equivalent. 
We conclude this work, in $\S 5$, with a collection of results concerning particular cases.

This paper is a condensed version of the author's doctoral dissertation at Universidade de São Paulo, Brasil passed on July 1977. The author is deeply grateful to his research adviser Professor Leopoldo Nachbin for the constant encouragement and help during the investigation and preparation of this thesis. Special thanks are due to Professors Mario Carvalho Matos and Jorge Mujica for their advice, help and stimulating discussions on my thesis subject.

\section{§ 0. Preliminaries}

We shall use freely the notations and terminology of [BMN] and [N1]. Unless stated otherwise, we shall adhere to the following conventions. $E$ and $F$ denote complex locally convex spaces and $U$ is a non-void open subset of $E$. The set of all continuous seminorms on $E$ is denoted by $C S(E)$; if $\alpha \in C S(E), r$ is a positive real number and $\xi \in E$, we denote by $B_{\alpha, r}(\xi)$ (resp. $\bar{B}_{\alpha, r}(\xi)$ ) the open (resp. closed) $\alpha$-ball of center $\xi$ and radius $r$ in $E$. We denote by $E_{\alpha}$ the vector space $E$ seminormed by $\alpha \in C S(E)$, by $E / \alpha$ the normed space associated with $E_{\alpha}$ and by $\dot{\alpha}$ the norm on $E / \alpha$.

If $I$ is a set and $F$ is a seminormed space, we denote by $\ell^{\infty}(I ; F)$ the seminormed space of all bounded mappings of $I$ into $F$.

We denote by $\mathscr{H}(U ; F)$ the vector space of all holomorphic mappings of $U$ into $F$ and by $H(U ; F)$ the space $\mathscr{H}(U ; \hat{F}) \cap F^{U}$, where $\hat{F}$ denotes a fixed completion of $F$.

A mapping $f: U \rightarrow F$ is said to be algebraically holomorphic if the restriction $f \mid U \cap S$ is holomorphic for all finite dimensional subspaces $S$ intersecting $U$, where $S$ is endowed with its natural (Hausdorff) topology. We denote by $\mathscr{H}_{a}(U ; F)$ the vector space of all algebraically holomorphic mappings of $U$ into $F$, by $\mathscr{H}_{c}(U ; F)$ the vector subspace of $\mathscr{H}_{a}(U ; F)$ of those mappings which are bounded on the compact subsets of $U$ and by $H_{c}(U ; F)$ the vector space $\mathscr{H}_{c}(U ; \hat{F}) \cap F^{U}$. We denote by $\mathscr{H}_{h}(U ; F)$ the vector subspace of $\mathscr{H}_{c}(U ; F)$ of those mappings which are hypoholomorphic, that is, algebraically holomorphic mappings which are continuous on the compact subsets of $U$.

If $m \in N$, we denote by $\mathscr{P}_{a}\left({ }^{m} E ; F\right)$ the vector space of all $m$-homogeneous polynomials of $E$ into $F$. We denote by $\mathscr{P}_{c}\left({ }^{m} E ; F\right)$ the vector subspace of $\left.\mathscr{P}_{a}{ }^{m} E ; F\right)$ of those polynomials which are bounded on the compact subsets 
(or equivalently, on the bounded subsets) of $E$. We denote by $\mathscr{P}_{h}\left({ }^{m} E ; F\right)$ the vector subspace of $\mathscr{P}_{c}\left({ }^{m} E ; F\right)$ of those polynomials which are hypoholomorphic, that is, $m$-homogeneous polynomials of $E$ into $F$ which are continuous on the compact subsets of $E$. We denote by $\mathscr{P}\left({ }^{m} E ; F\right)$ the vector space of all continuous $m$-homogeneous polynomials of $E$ into $F$.

If $m \in N$, we denote by $\left.\mathscr{L}_{a s}{ }^{m} E ; F\right)$ the vector space of all symmetric $m$-linear mappings of $E$ into $F$ and by $\left.\mathscr{L}_{s}{ }^{m} E ; F\right)$ the vector subspace of $\left.\mathscr{L}_{a s}{ }^{m} E ; F\right)$ of all continuous symmetric $m$-linear mappings of $E$ into $F$.

If $f \in \mathscr{H}(U ; F)$, we use the usual notations for the differential operators defined by the coefficients of the Taylor series of $f$ in points of $U$ :

$$
\begin{aligned}
& \hat{d}^{m}: f \in \mathscr{H}(U ; F) \longmapsto \hat{d}^{m} f \in \mathscr{H}\left(U ; \mathscr{P}\left({ }^{m} E ; F\right)\right) \\
& \left.d^{m}: f \in \mathscr{H}(U ; F) \longmapsto d^{m} f \in \mathscr{H}\left(U ; \mathscr{L}{ }^{m}{ }^{m} E ; F\right)\right) \quad(m \in N) .
\end{aligned}
$$

When $f \in \mathscr{H}_{a}(U ; F)$, we use the notation $\hat{\partial}^{m} f$ (resp. $\partial^{m} f$ ) instead of $\hat{d}^{m} f$ (resp. $d^{m} f$ ).

Given a space $\mathscr{S}$ of mappings of $U$ into $F$ which are bounded on the compact (resp. finite dimensional compact) subsets of $U$, we denote by $\mathscr{T}_{0}$ (resp. $\mathscr{T}_{\text {of }}$ ) the topology on $\mathscr{S}$ of uniform convergence on compact (resp. finite dimensional compact) subsets of $U$. If $\beta \in C S(F)$ and $K$ is a compact (resp. finite dimensional compact) subset of $U$, the $\mathscr{T}_{0}$-continuous (resp. $\mathscr{T}_{0 f}$-continuous) seminorm on $\mathscr{S}$ defined by $\beta$ and $K$ is denoted by $\|\cdot\|_{\beta, K}$, that is,

$$
\|f\|_{\beta, K}=\sup \{\beta(f(x)) \mid x \in K\} .
$$

A mapping $f: U \rightarrow F$ is amply bounded if $\beta \circ f$ is locally bounded for every $\beta \in C S(F)$; more generally, a collection $\mathscr{X}$ of mappings of $U$ into $F$ is amply bounded if the collection $\beta \circ \mathscr{X}$ is locally bounded for every $\beta \in$ $C S(F)$. We denote by $\mathscr{A} \mathscr{B}(U ; F)$ the set of all amply bounded subsets of $F^{u}$.

When $F=C$ we let $\mathscr{H}(U)$ stand for $\mathscr{H}(U ; C), \mathscr{P}\left({ }^{m} E\right)$ stand for $\mathscr{P}\left({ }^{m} E ; C\right)$ and $\mathscr{A} \mathscr{B}(U)$ stand for $\mathscr{A} \mathscr{B}(U ; C)$, etc.

The closed absolutely convex hull of a subset $X$ of $E$ is denoted by $\bar{\Gamma}_{a}(X)$.

A locally convex space $E$ is said to be holomorphically bornological if, for every $U$ and every $F$, we have $\mathscr{H}(U ; F)=\mathscr{H}_{c}(U ; F)$.

A locally convex space $E$ is said to be holomorphically barreled (resp. holomorphically infrabarreled) if, for every $U$ and every $F$, each collec- 
tion $\mathscr{X} \subset \mathscr{H}(U ; F)$ is amply bounded if (and always only if) $\mathscr{X}$ is $\mathscr{T}_{0 f^{-}}$ bounded (resp. $\mathscr{T}_{0}$-bounded).

A locally convex space $E$ is said to be holomorphically Mackey if, for every $U$ and every $F$, each mapping $f: U \rightarrow F$ belongs to $H(U ; F$ ) if (and always only if) $f$ is weakly holomorphic, that is, $\psi \circ f \in \mathscr{H}(U)$ for every $\psi$ $\in F^{\prime}$.

We use the following abbreviations of properties of a complex locally convex space: $\mathrm{B}=$ Baire, $\mathrm{S}=$ Silva, $\mathrm{sm}=$ semimetrizable, $\mathrm{hbo}=$ holomorphically bornological, hba = holomorphically barreled, hib $=$ holomorphically infrabarreled, $\mathrm{hM}=$ holomorphically Mackey. We have the following implications for the above properties, which we shall use freely (see $[\mathrm{BMN}]$ ):

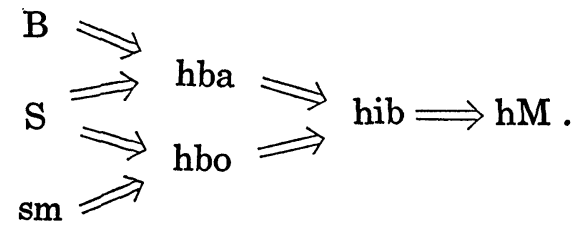

\section{§1. Polynomial classification of locally spaces. D-holomorphic and $D^{*}$-holomorphic mappings}

In this section we introduce the polynomially significant properties, properties $(D)$ and $\left(D^{*}\right)$, hypoholomorphic and holomorphically semibornological spaces. We shall show that the holomorphically significant properties can be expressed in terms of the polynomially significant properties and properties $(D)$ and $\left(D^{*}\right)$.

Definition 1.1. A mapping $f \in \mathscr{H}_{c}(U ; F)$ is said to be $D$-holomorphic if $\hat{\partial}^{m} f(\xi) \in \mathscr{P}\left({ }^{m} E ; F\right)$ for all $m \in N$ and all $\xi \in U$. We denote by $\mathscr{H}_{D}(U ; F)$ the vector space of all $D$-holomorphic mappings of $U$ into $F$. A locally convex space $E$ has the property $(D)$ if

$$
\mathscr{H}(U ; F)=\mathscr{H}_{D}(U ; F)
$$

for every $U$ and every $F$.

We shall use the abbreviation: $D=$ property $(D)$.

Definition 1.2. $E$ is a hypoholomorphic space if, for every $U$ and every $F$, the identity 


$$
\mathscr{H}(U ; F)=\mathscr{H}_{h}(U ; F)
$$

holds. $E$ is a polynomially hypoholomorphic space if, for every $m \in N$ and every $F$, the identity

$$
\mathscr{P}\left({ }^{m} E ; F\right)=\mathscr{P}_{h}\left({ }^{m} E ; F\right)
$$

holds.

We shall use the abbreviations: $\mathrm{h}=$ hypoholomorphic and $\mathrm{ph}=$ polynomially hypoholomorphic.

Proposition 1.3. For every $U$ and every $F$, we have

$$
\mathscr{H}(U ; F) \subset \mathscr{H}_{D}(U ; F) \subset \mathscr{H}_{n}(U ; F) \subset \mathscr{H}_{c}(U ; F) .
$$

Hence, we have the following implications between the properties $h b o, h$ and $D$ :

$$
h b o \Longrightarrow h \Longrightarrow D \text {. }
$$

Proof. It is enough to show that $\mathscr{H}_{D}(U ; F) \subset \mathscr{H}_{h}(U ; F)$. Given $f \in$ $\mathscr{H}_{D}(U ; F)$, a compact set $K \subset U$ and $\xi \in K$ we prove that $f \mid K$ is continuous at $\xi$. We first assume that $F$ is a Hausdorff space. Fix $\rho>1$, there exists $\alpha \in C S(E)$ such that $V=B_{\alpha, 1 / \rho}(\xi) \subset U,(1-\lambda) \xi+\lambda x \in U$ for all $\lambda \in C$ such that $|\lambda| \leq \rho$ and all $x \in V$. If $\beta \in C S(E)$, by Taylor's remainder formula, for all $x \in V \cap K$ and all $m \in N$,

$$
\beta(f(x)-f(\xi)) \leq \beta\left[\sum_{j=1}^{m} \frac{1}{j !} \hat{\partial}^{\jmath} f(\xi)(x-\xi)\right]+\frac{M}{\rho^{m}(\rho-1)}
$$

where

$$
M=\sup \{\beta(f((1-\lambda) \xi+\lambda x)) \mid x \in K \text { and }|\lambda|=\rho\} .
$$

Hence, $f$ is continuous since $\rho>1$. For an arbitrary $F$, let $\tilde{F}$ be the Hausdorff space associated with $F$ and let $q: F \rightarrow \tilde{F}$ be the quotient mapping. By the preceding case, we have $\mathscr{H}_{D}(U ; \tilde{F}) \subset \mathscr{H}_{h}(U ; \tilde{F})$. Given $f \in$ $\mathscr{H}_{D}(U ; F)$ it is clear that $q \circ f \in \mathscr{H}_{D}(U ; \tilde{F})$, hence $q \circ f \in \mathscr{H}_{h}(U ; \tilde{F})$ which implies that $f \in \mathscr{H}_{h}(U ; F)$. In fact, fix a compact set $K \subset U, \xi \in K$ and a closed neighborhood $V$ of $f(\xi)$ in $F, V_{o}=q(V)$ is a neighborhood of $q(f(\xi))$, hence we can find a neighborhood $W$ of $\xi$ in $K$ such that $(q \circ f)(W) \subset V_{o}$, whence $f(W) \subset \bar{V}=V$.

Remark. If a complex locally convex space $E$ is a $k$-space then $E$ is hypoholomorphic and hence has the property $(D)$. 
Proposition 1.4. If $E$ is a hypoholomorphic space, then $\left(\mathscr{H}(U ; F) ; \mathscr{T}_{0}\right)$ is complete, if $F$ is complete.

Proof. If $F$ is complete, the space $\left(\mathscr{H}_{c}(U ; F) ; \mathscr{T}_{0}\right)$ is complete. Since $\mathscr{H}_{h}(U ; F)$ is closed in $\left(\mathscr{H}_{c}(U ; F) ; \mathscr{T}_{0}\right)$ it follows that $\left(\mathscr{H}_{h}(U ; F) ; \mathscr{T}_{0}\right)$ is complete. Since $E$ is a hypoholomorphic space we have $\mathscr{H}(U ; F)=\mathscr{H}_{h}(U ; F)$.

Definition 1.5. A given $E$ is said to be a polynomially bornological space if, for every $m \in N$ and every $F$, the identity

$$
\left.\mathscr{P}\left({ }^{m} E ; F\right)=\mathscr{P}_{c}{ }^{m} E ; F\right)
$$

holds.

We shall use the abbreviation: pbo = polynomially bornological.

Proposition 1.6. For a given $E$ the following conditions are equivalent:

(i) $E$ is holomorphically bornological;

(ii) $E$ is hypoholomorphic and polynomially bornological;

(iii) E has property $(D)$ and is polynomially bornological.

Proof. Clearly, every holomorphically bornological space is polynomially bornological, hence Proposition 1.3. implies that (i) $\Rightarrow$ (ii) $\Rightarrow$ (iii). We shall prove that (iii) $\Rightarrow$ (i). Given $U$ and $f \in \mathscr{H}_{c}(U ; F)$, where we assume that $F$ is Hausdorff, if $K$ is a compact subset of $E$ and $\xi \in U$, there is $\rho$ $>0$ such that $\xi+\lambda x \in U$ for all $\lambda \in C$ such that $|\lambda| \leq \rho$ and all $x \in K$. Hence by Cauchy's integral formula, we have for $m \in N$

$$
\hat{\partial}^{m} f(\xi)(K) \subset \frac{m !}{\rho^{m}} \bar{\Gamma}_{a}\{f(\xi+\lambda x) \mid x \in K \text { and }|\lambda|=\rho\} .
$$

Since $E$ is polynomially bornological, it follows that

$$
\hat{\partial}^{m} f(\xi) \in \mathscr{P}\left({ }^{m} E ; F\right)
$$

for all $m \in N$ and all $\xi \in U$, which implies that $f \in \mathscr{H}(U ; F)$, since $E$ has the property $(D)$. The case of an arbitrary $F$ follows from this as in Proposition 1.3.

Proposition 1.7. For a locally convex space $E$ the following conditions are equivalent:

(i) $E$ is hypoholomorphic;

(ii) E has property (D) and is polynomially hypoholomorphic.

Proof. The implication (i) $\Rightarrow$ (ii) follows from Proposition 1.3. To show 
that (ii) $\Rightarrow$ (i), given $U$ and $f \in \mathscr{H}_{h}(U ; F)$, where we suppose that $F$ is Hausdorff, if $\xi \in U$ and $K$ is a compact subset of $E$, there is $\rho>0$ such that $\xi+\lambda x \in U$ for all $\lambda \in C$ such that $|\lambda| \leq \rho$ and all $x \in K$. Hence by Cauchy's integral formula, if $m \in N, \beta \in C S(F)$ and $\eta \in K$, for all $x \in K$ we have

$$
\beta\left(\hat{\partial}^{m} f(\xi)(x)-\hat{\partial}^{m} f(\xi)(\eta)\right) \leq \frac{m !}{\rho^{m}} \sup \{\beta(f(\xi+\lambda x)-f(\xi+\lambda \eta))|| \lambda \mid=\rho\} .
$$

Since

$$
L=\{\xi+\lambda x \mid x \in K \text { and }|\lambda|=\rho\}
$$

is a compact subset of $U$, it follows that $f \mid L$ is uniformly continuous, hence for a given $\varepsilon>0$ there is $\alpha \in C S(E)$ such that

$$
y, z \in L \quad \text { and } \quad \alpha(y-z) \leq 1 \Longrightarrow \beta(f(y)-f(z))<\frac{\varepsilon \rho^{m}}{m !} .
$$

Hence, for all $x \in B_{\alpha, 1 / \rho}(\eta) \subset K$ we have

$$
\beta\left(\hat{\partial}^{m} f(\xi)(x)-\hat{\partial}^{m} f(\xi)(\eta)\right) \leq \varepsilon
$$

which proves that

$$
\hat{\partial}^{m} f(\xi) \in \mathscr{P}\left({ }^{m} E ; F\right), \quad \text { for all } m \in N \text { and all } \xi \in U,
$$

since $E$ is polynomially hypoholomorphic. The property $(D)$ of $E$ implies then $f \in \mathscr{H}(U ; F)$. The case of an arbitrary $F$ follows from this.

Definition 1.8. A given $E$ is said be a polynomially infrabarreled space if, for all $m \in N$ and all $F$, each collection $\mathscr{X} \subset \mathscr{P}\left({ }^{m} E ; F\right)$ which is bounded on all compact subsets of $E$ is equicontinuous (or equivalently, amply bounded).

We shall use the abbreviation: pib = polynomially infrabarreled.

Lemma 1.9. Let $E$ be a polynomially infrabarreled space and let $\mathscr{X} \subset$ $\mathscr{H}(U ; F)$ be a collection bounded on all compact subsets of $U$. Then, for all $\xi \in U$ and all $m \in N$, the set

$$
\mathscr{X}_{m, \xi}=\left\{\hat{d}^{m} f(\xi) \mid f \in \mathscr{X}\right\}
$$

is amply bounded.

Proof. It is enough to show that $\mathscr{X}_{m, \xi}$ is bounded on all compact subsets of $E$. Let $K$ be a compact set in $E$, then there exists $\rho>0$ such 
that $\xi+\lambda x \in U$ for all $\lambda \in C$ such that $|\lambda| \leq \rho$ and all $x \in K$. Hence by Cauchy's integral formula, we have

$$
\hat{d}^{m} f(\xi)(K) \subset \frac{m !}{\rho^{m}} B_{f}
$$

where

$$
B_{f}=\bar{\Gamma}_{a}\{f(\xi+\lambda x)|| \lambda \mid=\rho \text { and } x \in K\}
$$

It follows that

$$
\bigcup_{f \in \mathscr{x}} \hat{d}^{m} f(\xi)(K) \subset \frac{m !}{\rho^{m}} \bigcup_{f \in \mathscr{x}} B_{f} .
$$

Since $\mathscr{X}$ is bounded on the compact set

$$
\{\xi+\lambda x|| \lambda \mid=\rho \text { and } x \in K\} \text {. }
$$

the set

$$
X=\bar{\Gamma}_{a}\{f(\xi+\lambda x)|| \lambda \mid=\rho, x \in K \text { and } f \in \mathscr{X}\}
$$

is bounded in $F$, thus the relation

$$
\bigcup_{f \in \mathscr{x}} B_{f} \subset X
$$

implies that

$$
\bigcup_{f \in \mathscr{x}} \hat{d}^{m} f(\xi)(K)=\left\{\hat{d}^{m} f(\xi)(x) \mid x \in K \text { and } f \in \mathscr{X}\right\} \subset \frac{m !}{\rho^{m}} X .
$$

Proposition 1.10. For a locally convex space $E$ the following conditions are equivalent:

(i) $E$ is holomorphically infrabarreled;

(ii) $E$ has property (D) and is polynomially infrabarreled.

Proof. (i) $\Rightarrow$ (ii): It is sufficient to prove that $E$ has property $(D)$. Given $U, \xi \in U$ and $f \in \mathscr{H}_{D}(U ; F)$ where we assume that $F$ is Hausdorff, we shall prove that there exists a neighborhood $V$ of $\xi$ in $U$ such that $f \mid V$ is continuous. Fix $\rho>1$, then there is $\alpha \in C S(E)$ such that $V=B_{\alpha, 1 / \rho}(\xi)$ $\subset U,(1-\lambda) \xi+\lambda x \in U$, for all $\lambda \in C$ such that $|\lambda| \leq \rho$ and all $x \in V$ and

$$
f(x)-\sum_{j=0}^{m} \frac{1}{j !} \hat{\partial}^{\jmath} f(\xi)(x-\xi)=\frac{1}{2 \pi i} \int_{|\lambda|=\rho} \frac{f((1-\lambda) \xi+\lambda x)}{\lambda^{m+1}(\lambda-1)} d \lambda,
$$


for every $x \in V$ and every $m \in N$. Hence the set $\mathscr{X}=\left\{f_{m} \mid m \in N\right\}$, where for all $m \in N$ we set

$$
f_{m}: x \in V \longmapsto \sum_{j=0}^{m} \frac{1}{j !} \hat{\partial}^{j} f(\xi)(x-\xi) \in F,
$$

is bounded in $\left(\mathscr{H}(V ; F) ; \mathscr{T}_{0}\right)$. Since $E$ is holomorphically infrabarreled, it follows that $\mathscr{X}$ is equicontinuous. Since $\lim _{m \rightarrow \infty} f_{m}(x)=f(x)$ for every $x \in V$, the set $\mathscr{X}(x)=\left\{f_{m}(x) \mid m \in N\right\}$ is relatively compact in $F$ for all $x \in V$. Hence, by Ascoli's theorem, $\mathscr{X}$ is a relatively compact set in $\left(\mathscr{C}(V ; F) ; \mathscr{T}_{0}\right)$. It follows that the elementary filter associated with the sequence $\left(f_{m}\right)_{m \in N}$ has an adherent point $g \in \mathscr{C}(V ; F)$, which implies that

$$
\beta(f(x)-g(x))=0 \quad \text { for all } x \in V \text { and all } \beta \in C S(F) .
$$

Since $F$ is Hausdorff, it follows that $f \mid V=g$, hence $f \in \mathscr{H}(U ; F)$. The case of an arbitrary $F$ follows from this.

(ii) $\Rightarrow($ i $)$ : Let $\mathscr{X}$ be a bounded set in $\left(H(U) ; \mathscr{T}_{0}\right)$, we shall show that $\mathscr{X}$ is locally bounded. Consider the mapping

$$
f_{x}: x \in U \longmapsto(g(x))_{g \in \mathscr{x}} \in \ell^{\infty}(\mathscr{X}) .
$$

It is known that

$$
f_{x} \in \mathscr{H}_{c}\left(U ; \ell^{\infty}(\mathscr{X})\right)
$$

Since $E$ is polynomially infrabarreled, Lemma 1.9 implies that $\hat{\partial}^{m} f_{x}(\xi)$ is locally bounded, hence continuous, for every $\xi \in U$ and every $m \in N$, which proves that

$$
f_{\mathscr{x}} \in \mathscr{H}_{D}\left(U ; \ell^{\infty}(\mathscr{X})\right)
$$

Property $(D)$ of $E$ then implies that $f_{\mathscr{x}} \in \mathscr{H}\left(U ; \ell^{\infty}(\mathscr{X})\right)$, thus $f_{\mathscr{x}}$ is locally bounded, that is, $\mathscr{X}$ is locally bounded.

CoRollary 1.11. For a polynomially bornological space $E$ the following conditions are equivalent:

(i) $E$ is holomorphically bornological;

(ii) $E$ is holomorphically infrabarreled;

(iii) $E$ is hypoholomorphic;

(iv) $E$ has property (D).

Proof. In view of Proposition 1.6 we have (i) $\Leftrightarrow$ (iii) $\Leftrightarrow$ (iv). The implication (ii) $\Rightarrow$ (iv) follows from Proposition 1.10 and it is known that (i) $\Rightarrow$ (ii). 
Now we shall prove a statement corresponding to Proposition 1.10 for holomorphically barreled spaces. We shall need the following definitions.

Definition 1.12. A given $E$ is said to be a polynomially barreled space if, for all $m \in N$ and all $F$, each collection $\mathscr{X} \subset \mathscr{P}\left({ }^{m} E ; F\right)$ which is bounded on all finite dimensional compact subsets of $E$ is equicontinuous (or equivalently, amply bounded).

We shall use the abbreviation: pba $=$ polynomially barreled.

Definition 1.13. A mapping $f \in \mathscr{H}_{a}(U ; F)$ is said to be $D^{*}$-holomorphic if $\hat{\partial}^{m} f(\xi) \in \mathscr{P}\left({ }^{m} E ; F\right)$ for all $m \in N$ and all $\xi \in U$. We denote by $\mathscr{H}_{D^{*}}(U ; F)$ the vector space of all $D^{*}$-holomorphic mappings of $U$ into $F$. A locally convex space $E$ has property $\left(D^{*}\right)$ if

$$
\mathscr{H}(U ; F)=\mathscr{H}_{D^{*}}(U ; F)
$$

for every $U$ and every $F$.

We shall use the abbreviation: $D^{*}=$ property $\left(D^{*}\right)$

Clearly we have: $D^{*} \Longrightarrow D$.

LEMMa 1.14. Let $E$ be a polynomially barreled space and let $\mathscr{X} \subset$ $\mathscr{H}(U ; F)$ be a collection bounded on all finite dimensional compact subsets of $U$. Then, for all $\xi \in U$ and all $m \in N$, the set

$$
\mathscr{X}_{m, \xi}=\left\{\hat{d}^{m} f(\xi) \mid f \in \mathscr{X}\right\}
$$

is amply bounded.

Proof. The argument is a minor modification of the proof of Lemma 1.9 .

Proposition 1.15. For a given $E$ the following conditions are equivalent:

(i) $E$ is holomorphically barreled;

(ii) $E$ has property $\left(D^{*}\right)$ and is polynomially barreled.

Proof. The argument is similar to the proof of Proposition 1.10.

Definition 1.16. A given $E$ is said to be a strongly polynomially barreled space, if for all $m \in N$ and all $F$, each collection $\mathscr{X} \subset \mathscr{P}\left({ }^{m} E ; F\right)$ which is bounded on all finite subsets of $E$ is equicontinuous (or equivalently, amply bounded).

We shall use the abbreviation: spba $=$ strongly polynomially barreled.

Clearly we have: $\mathrm{spba} \Longrightarrow$ pba . 
The following result furnishes a class of polynomially bornological spaces and a class of strongly polynomially barreled spaces.

Proposition 1.17. (a) If $E$ is barreled, bornological and (DF), then $E$ is polynomially bornological.

(b) If $E$ is barreled and $(D F)$, then $E$ is strongly polynomially barreled and hence polynomially barreled.

Proof. (a) Given $F, m \in N$ and $P \in \mathscr{P}_{c}\left({ }^{m} E ; F\right)$, there exists $A \in$ $\left.\mathscr{L}_{a s}{ }^{m} E ; F\right)$ such that $\hat{A}=P$. We shall prove that $A$ is continuous. Since $E$ is $(D F)$ and barreled, it is enough to show (see [G], Ch. IV, $\S 3$, No. 2, Corollary 1 of Theorem 2) that $A$ is separately continuous and since $A$ is symmetric, it suffices to show that $A$ is continuous in the first variable. That is, fix $a_{2}, \cdots, a_{m}$ in $E$ then we must show that the linear mapping

$$
u: x \in E \longmapsto A\left(x, a_{2}, \cdots, a_{m}\right) \in F
$$

is continuous. Since $E$ is bornological, it is enough to prove that $u$ is bounded, which follows from the polarization formula and the fact that $P$ is bounded on bounded sets.

(b) Given $F, m \in N$ and a collection $\hat{\mathscr{X}} \subset \mathscr{P}\left({ }^{m} E ; F\right)$ such that $\hat{\mathscr{X}}$ is bounded on all finite subsets of $E$, we shall show that $\hat{\mathscr{X}}$ is equicontinuous. Consider the set

$$
\mathscr{X}=\left\{A \in \mathscr{L}_{s}\left({ }^{m} E ; F\right) \mid \hat{A} \in \hat{\mathscr{X}}\right\} .
$$

Then, by the polarization formula and since $\hat{\mathscr{X}}$ is bounded on all finite subsets of $E$, it follows that $\mathscr{X}$ is bounded in $\mathscr{L}_{s}\left({ }^{m} E ; F\right)$ for the topology of simple convergence. Therefore (see loc. cit.), $\mathscr{X}$ is equicontinuous hence $\hat{\mathscr{X}}$ is equicontinuous.

Definition 1.18. $E$ is said to be a locally holomorphically infrabarreled space if, for all $U$, all $F$ and all collections $\mathscr{X} \subset \mathscr{H}(U ; F)$ which are bounded on the compact subsets of $U$, the set

$$
\mathscr{X} \mid K=\{g|K| g \in \mathscr{X}\}
$$

is an equicontinuous subset of $\mathscr{C}(K ; F)$ for every compact subset $K$ of $U$. We shall use the abbreviation: lhib = locally holomorphically infrabarreled.

Proposition 1.19. For a given $E$ the following conditions are equivalent:

(i) $E$ is locally holomorphically infrabarreled;

(ii) $E$ satisfies Definition 1.18 in the case $F=C$; 
(iii) For all $U$, a collection $\mathscr{X} \subset \mathscr{H}(U)$ is $\mathscr{T}_{0}$-bounded if and only if $\mathscr{X}$ is $\mathscr{T}_{0}$-precompact.

Proof. Clearly (i) $\Rightarrow$ (ii). We shall prove that (ii) $\Rightarrow$ (i). Given $U$ and $F$, let $\mathscr{X}$ be a bounded subset of $\left(\mathscr{H}(U ; F) ; \mathscr{T}_{0}\right)$. Fix a compact set $K \subset$ $U, \beta \in C S(F)$ and consider the set

$$
\Psi=\left\{\psi \in\left(F_{\beta}\right)^{\prime} \mid\|\psi\|_{\beta}=1\right\} .
$$

Then, is clear that the set

$$
\mathscr{X}(\Psi)=\{\psi \circ f \mid f \in \mathscr{X} \text { and } \psi \in \Psi\}
$$

is a bounded subset of $\left(\mathscr{H}(U) ; \mathscr{T}_{0}\right)$. By (ii) it follows that

$$
\mathscr{X}(\Psi) \mid K=\{h|K| h \in \mathscr{X}(\Psi)\}
$$

is an equicontinuous subset of $\mathscr{C}(K)$. Hence, for $\xi \in K$, there exists a neighborhood $V$ of $\xi$ in $K$ such that

$$
|g(x)-g(\xi)| \leq 1 \quad \text { for all } x \in V \text { and all } g \in \mathscr{X}(\Psi) \mid K .
$$

By the Hahn-Banach theorem we have

$$
\begin{aligned}
& \sup \{\beta(f(x)-f(\xi)) \mid f \in \mathscr{X} \text { and } x \in V\} \\
& \quad=\sup \{|\psi(f(x)-f(\xi))| \mid f \in \mathscr{X}, x \in V \text { and } \psi \in \Psi\},
\end{aligned}
$$

whence $\beta(f(x)-f(\xi)) \leq 1$ for all $x \in V$ and all $f \in \mathscr{X}$.

(ii) $\Rightarrow$ (iii): Let $\mathscr{X}$ be a bounded subset of $\left(\mathscr{H}(U) ; \mathscr{T}_{0}\right)$. By (ii), for every compact subset $K$ of $U$, the set

$$
\mathscr{X} \mid K=\{g|K| g \in \mathscr{X}\}
$$

is an equicontinuous subset of $\mathscr{C}(K)$ and, since the set

$$
(\mathscr{X} \mid K)(x)=\{g(x) \mid g \in \mathscr{X}\}
$$

is bounded for every $x \in K$, by Ascoli's theorem, $\mathscr{X} \mid K$ is a relatively compact subset of $\mathscr{C}(K)$. On the other hand, it is clear that $\mathscr{T}_{0}$ coincides with the projective topology on $\mathscr{H}(U)$ for the family of linear mappings

$$
P_{K}: f \in \mathscr{H}(U) \longmapsto f \mid K \in \mathscr{C}(K)
$$

where $K$ describes the set of all compact subsets of $U$. Therefore, since $p_{K}(\mathscr{X})=\mathscr{X} \mid K$ is a precompact subset of $\mathscr{C}(K)$ for every compact subset $K$ of $U$, it follows that $\mathscr{X}$ is a precompact subset of $\left(\mathscr{H}(U) ; \mathscr{T}_{0}\right)$.

(iii) $\Rightarrow$ (ii): Let $\mathscr{X}$ be a bounded subset of $\left(\mathscr{H}(U) ; \mathscr{T}_{0}\right)$. Then by (iii), 
$\mathscr{X}$ is a precompact subset of $\left(\mathscr{H}(U) ; \mathscr{T}_{0}\right)$, whence $\mathscr{X} \mid K=p_{K}(\mathscr{X})$ is a relatively compact subset of $\mathscr{C}(K)$ for every compact subset $K$ of $U$. By Ascoli's theorem, $\mathscr{X} \mid K$ is equicontinuous for every compact subset $K$ of $U$.

Proposition 1.20. If $E$ is hypoholomorphic and locally holomorphically infrabarreled, then $E$ is holomorphically infrabarreled.

Proof. Given $U$ and a bounded subset $\mathscr{X}$ of $\left(\mathscr{H}(U) ; \mathscr{T}_{0}\right)$, consider the mapping

$$
f_{x}: x \in U \longmapsto(g(x))_{g \in x} \in \ell^{\infty}(\mathscr{X}) .
$$

Since $E$ is locally holomorphically infrabarreled it follows that

$$
f_{x} \in \mathscr{H}_{h}\left(U ; \ell^{\infty}(\mathscr{X})\right)
$$

Hence

$$
f_{x} \in \mathscr{H}\left(U ; \ell^{\infty}(\mathscr{X})\right)
$$

since $E$ is hypoholomorphic. It now follows that $f_{x}$ is locally bounded, i.e. $\mathscr{X}$ is locally bounded.

Let $U$ be a non-void open set in a locally convex space $E$ and let $F$ be a locally convex space. We denote by

$$
\mathscr{H}_{s c}(U ; F)
$$

the complex vector space of all algebraically holomorphic mappings of $U$ into $F$ which are sequentially continuous. With this notation we set:

Definition 1.21. A given $E$ is said to be a holomorphically semibornological space if, for all $U$ and all $F$, the identity

$$
\mathscr{H}(U ; F)=\mathscr{H}_{s c}(U ; F)
$$

holds.

We shall use the abbreviation: hsb = holomorphically semibornological.

For given $E, F$ and $U$, we have $\mathscr{H}_{h}(U ; F) \subset \mathscr{H}_{s c}(U ; F)$. On the other hand, if every compact set in $E$ is sequentially compact, then $\mathscr{H}_{s c}(U ; F)$ $\subset \mathscr{H}_{c}(U ; F)$. Hence, by Proposition 1.3 , we get:

Proposition 1.22. Let $E$ be a locally convex space such that every compact set in $E$ is sequentially compact. Then, for all $U$ and $F$, we have

$$
\mathscr{H}(U ; F) \subset \mathscr{H}_{D}(U ; F) \subset \mathscr{H}_{h}(U ; F) \subset \mathscr{H}_{s c}(U ; F) \subset \mathscr{H}_{c}(U ; F) .
$$

As a consequence, in $E$, we have the following implications 


$$
h b o \Longrightarrow h s b \Longrightarrow h \Longrightarrow D \text {. }
$$

Proposition 1.23. Given $E, F$ and $U$, the following are true:

(a) If $f \in \mathscr{H}_{c}(U ; F)$, then $\hat{\partial}^{m} f(\xi) \in \mathscr{P}_{c}\left({ }^{m} E ; F\right)$ for all $m \in N$ and $\xi \in U$.

(b) If $E$ is polynomially bornological, we have

$$
\mathscr{H}_{D}(U ; F)=\mathscr{H}_{h}(U ; F)=\mathscr{H}_{c}(U ; F)
$$

(c) If $E$ is polynomially bornological and every compact set in $E$ is sequentially compact, we have

$$
\mathscr{H}_{D}(U ; F)=\mathscr{H}_{h}(U ; F)=\mathscr{H}_{s c}(U ; F)=\mathscr{H}_{c}(U ; F) .
$$

Proof. The first assertion is contained in the proof of Proposition 1.6 and the second assertion follows from (a) and Proposition 1.3. The last assertion follows from (b) and Proposition 1.22.

Proposition 1.24. For a polynomially bornological space $E$ the following conditions are equivalent:

(i) $E$ is holomorphically bornological;

(ii) $E$ is holomorphically infrabarreled;

(iii) $E$ is hypoholomorphic;

(iv) $E$ has property (D).

If every compact set in $E$ is sequentially compact, the above conditions are equivalent to the following one:

(v) $E$ is holomorphically semibornological.

Proof. Apply Corollary 1.11, and Proposition 1.23 (c).

ExAmple. Let $X$ be an infinite dimensional complex Banach space, let $Y=C^{(N)}$ be endowed with the locally convex direct sum topology and let $E=X \times Y$ be the locally convex product space. Since $E$ is barreled, bornological and $(D F)$, Proposition 1.17 implies that $E$ is polynomially bornological and strongly polynomially barreled (hence polynomially barreled). On the other hand (see [BMN], Example 18) $E$ is not holomorphically bornological. Thus we have the following:

(a) $E$ is a polynomially bornological space which is not a holomorphically bornological space. By Proposition 1.6 it follows that $E$ does not have property $(D)$.

(b) $E$ has not property $\left(D^{*}\right)$, since $D^{*} \Rightarrow D$.

(c) $E$ is not a holomorphically infrabarreled space, by Proposition 1.10 . 
(d) $E$ is a strongly polynomially barreled space which is not holomorphically barreled (by (b) and Proposition 1.15).

(e) $E$ is a polynomially infrabarreled space which is not holomorphically infrabarreled.

Remark. If $E$ is a holomorphically infrabarreled space which is not a holomorphically bornological space, then, by Proposition 1.6 and Proposition $1.10, E$ has property $(D)$ and it is not a polynomially bornological space.

\section{§2. Holomorphical classification and approximation properties}

The starting point of this section is the proof, with some hypothesis, that the completion of $\left(\mathscr{H}(U ; F) ; \mathscr{T}_{0}\right)$ is $\left(\mathscr{H}_{h}(U ; F) ; \mathscr{T}_{0}\right)$ for every $\xi$ equilibrated open subset $U$ of $E$ and every complete Hausdorff locally convex space $F$. We apply these results to find, for polynomially bornological spaces, new equivalent conditions involving holomorphically significant properties and completeness of $\left(\mathscr{H}(U ; F) ; \mathscr{T}_{0}\right)$. In the sequel, we denote by

$$
\left(\mathscr{H}(U ; F) ; \mathscr{T}_{0}\right)^{\wedge}
$$

the completion of the locally convex space $\left(\mathscr{H}(U ; F) ; \mathscr{T}_{0}\right)$.

Lemma 2.1. Let $U$ be a $\xi$-equilibrated open subset of a locally convex space $E$ and let $F$ be a Hausdorff locally convex space. We suppose that the following condition is satisfied:

For every $m \in N$, every $P \in \mathscr{P}_{h}\left({ }^{m} E ; F\right)$, every $\beta \in C S(F)$, every compact

(*) subset $K$ of $E$ and every $\varepsilon>0$, there exists $f \in \mathscr{H}(E ; F)$ such that $\|P-f\|_{\beta, K} \leq \varepsilon$.

Then, $\mathscr{H}(E ; F)$ and $\mathscr{H}(U ; F)$ are dense in $\left(\mathscr{H}_{n}(U ; F) ; \mathscr{T}_{0}\right)$ and hence, if $F$ is complete, we have:

$$
\left(\mathscr{H}(U ; F) ; \mathscr{T}_{0}\right)^{\wedge}=\left(\mathscr{H}_{h}(U ; F) ; \mathscr{T}_{0}\right) .
$$

Proof. Given $g \in \mathscr{H}_{h}(U ; F)$, a compact subset $K$ of $U, \beta \in C S(F)$ and $\varepsilon>0$, since $U$ is $\xi$-equilibrated, for all $x \in U$ we have

$$
g(x)=\sum_{m=0}^{\infty} \frac{1}{m !} \hat{\partial}^{m} g(\xi)(x-\xi), \quad \text { uniformly on } K .
$$

Hence there exists $\nu \in N$ such that 


$$
\beta\left(g(x)-\sum_{m=0}^{\nu} \frac{1}{m !} \hat{\partial}^{m} g(\xi)(x-\xi)\right) \leq \varepsilon / 2, \quad \text { for all } x \in K
$$

Let $\delta$ be a real number such that $0<\delta<1$ and $\delta(1-\delta)^{-1}<\varepsilon / 2$. Since $K-\xi=\{x-\xi \mid x \in K\}$ is compact in $E$ and, by Cauchy's integral formula we have

$$
\hat{\partial}^{m} g(\xi) \in \mathscr{P}_{h}\left({ }^{m} E ; F\right) \quad \text { for all } m \in N,
$$

the hypothesis $\left(^{*}\right)$ implies that there exists a finite sequence $\left(f_{m}\right)_{0 \leq m \leq \nu}$ in $\mathscr{H}(E ; F)$ such that

$$
\begin{aligned}
& \left\|f_{m}-\frac{1}{m !} \hat{\partial}^{m} g(\xi)\right\|_{\beta, K-\xi} \leq \delta^{m}, \quad \text { for all } m=1,2, \cdots, \nu, \\
& f_{0}: x \in E \longmapsto g(\xi) \in F .
\end{aligned}
$$

Clearly, the mapping

$$
f: x \in E \longmapsto \sum_{m=0}^{\nu} f_{m}(x-\xi) \in F
$$

is holomorphic and it is easy to see that

$$
\|g-f\|_{\beta, K} \leq \varepsilon
$$

which proves that $\mathscr{H}(E ; F)$ is dense in $\left(\mathscr{H}_{h}(U ; F) ; \mathscr{T}_{0}\right)$, hence $\mathscr{H}(U ; F)$ is also dense in $\left(\mathscr{H}_{h}(U ; F) ; \mathscr{T}_{0}\right)$. The proof of Proposition 1.4 shows that $\left(\mathscr{H}_{h}(U ; F) ; \mathscr{T}_{0}\right)$ is complete if $F$ is complete whence the statement about the completion follows.

CoRollary 2.2. Let $U$ be a $\xi$-equilibrated open subset of a locally convex space $E$ and let $F$ be a Hausdorff locally convex space. We suppose that the following condition is satisfied:

(*) $\quad \mathscr{P}\left({ }^{m} E ; F\right)$ is dense in $\left(\mathscr{P}_{h}\left({ }^{m} E ; F\right) ; \mathscr{T}_{0}\right)$ for all $m \in N$.

Then, $\mathscr{H}(E ; F)$ and $\mathscr{H}(U ; F)$ are dense in $\left(\mathscr{H}_{h}(U ; F) ; \mathscr{T}_{0}\right)$ and hence, if $F$ is complete, we have

$$
\left(\mathscr{H}(U ; F) ; \mathscr{T}_{0}\right)^{\wedge}=\left(\mathscr{H}_{h}(U ; F) ; \mathscr{T}_{0}\right) .
$$

Proof. In view of the hypothesis $(*)$ we can choose the sequence $f_{0}, f_{1}, \cdots, f_{\nu}$ which appear in the proof of Lemma 2.1 such that $f_{m} \in \mathscr{P}\left({ }^{m} E ; F\right)$ for all $m=0,1, \cdots, \nu$.

CoRollaRY 2.3. Let $U$ be a -equilibrated open subset of a polynomially 
hypoholomorphic space $E$ (see Definition 1.2) and let $F$ be a Hausdorff locally convex space. Then $\mathscr{H}(E ; F)$ and $\mathscr{H}(U ; F)$ are dense in $\left(\mathscr{H}_{h}(U ; F) ; \mathscr{T}_{0}\right)$ and hence, if $F$ is complete, we have

$$
\left(\mathscr{H}(U ; F) ; \mathscr{T}_{0}\right)^{\wedge}=\left(\mathscr{H}_{n}(U ; F) ; \mathscr{T}_{0}\right) \text {. }
$$

Proof. The hypothesis $(*)$ of Corollary 2.2 is trivially fulfilled in this case.

Since $\mathscr{P}\left({ }^{m} E ; F\right) \subset \mathscr{P}_{h}\left({ }^{m} E ; F\right) \subset \mathscr{P}_{c}\left({ }^{m} E ; F\right)$ for all $E, F$ and $m \in N$, we have pbo $\Rightarrow \mathrm{ph}$, hence from Corollary 2.3 we get:

Proposition 2.4. Let $U$ be a $\xi$-equilibrated open subset of a polynomially bornological space $E$ and let $F$ be a Hausdorff locally convex space. Then $\mathscr{H}(E ; F)$ and $\mathscr{H}(U ; F)$ are dense in $\left(\mathscr{H}_{h}(U ; F) ; \mathscr{T}_{0}\right)$ and hence, if $F$ is complete, we have

$$
\left(\mathscr{H}(U ; F) ; \mathscr{T}_{0}\right)^{\wedge}=\left(\mathscr{H}_{h}(U ; F) ; \mathscr{T}_{0}\right) .
$$

In the following two results, the expression

" $U$ is equilibrated"

signifies that there exists $\xi \in U$ such that $U$ is $\xi$-equilibrated.

Proposition 2.5. For a polynomially bornological space $E$, the following conditions are equivalent:

(i) $E$ is holomorphically bornological;

(ii) $\left(\mathscr{H}(U ; F) ; \mathscr{T}_{0}\right)$ is complete for every non-void open subset $U$ of $E$ and every complete Hausdorff locally convex space $F$;

(iii) $\left(\mathscr{H}(U ; F) ; \mathscr{T}_{0}\right)$ is complete for every equilibrated open subset $U$ of $E$ and every complete Hausdorff locally convex space $F$;

(iv) $E$ is holomorphically Mackey and $\left(\mathscr{H}(U) ; \mathscr{T}_{0}\right)$ is complete for every equilibrated open subset $U$ of $E$.

Proof. The implications (i) $\Rightarrow$ (ii) $\Rightarrow$ (iii) and (i) $\Rightarrow$ (iv) are trivial. We shall prove that (iii) $\Rightarrow$ (i). By Corollary 1.11 it is enough to show that $E$ is hypoholomorphic. Condition (iii) and Proposition 2.4 imply that

$$
\mathscr{H}(U ; F)=\mathscr{H}_{h}(U ; F)
$$

for every equilibrated open subset $U$ of $E$ and for every complete Hausdorff locally convex space $F$. This implies that (1) is valid for any non void open subset $U$ of $E$. So it remains to show only that (1) is valid for all 
$F$. Let $U$ and $F$ be arbitrary, let $\hat{F}$ be the Hausdorff completion of $F$ and let $j: F \rightarrow \hat{F}$ be the canonical map. Given $f \in \mathscr{H}_{h}(U ; F)$ it is clear that $j \circ f \in \mathscr{H}_{h}(U ; \hat{F})=\mathscr{H}(U ; \hat{F})$ and hence $f$ is amply bounded and therefore $f \in$ $\mathscr{H}(U ; F)$. Finally we shall prove that (iv) $\Rightarrow(\mathrm{i})$. The preceding argument show that $E$ is "scalarly hypoholomorphic" (that is, $E$ satisfies Definition 1.2 in the case $F=C$ ) since $\left(\mathscr{H}(U) ; \mathscr{T}_{0}\right)$ is complete for any equilibrated open subset $U$ of $E$. Hence, since $E$ is holomorphically Mackey it follows that $E$ is hypoholomorphic.

TheOREM 2.6. For a polynomially bornological space $E$, the following conditions are equivalent:

(i) $E$ is holomorphically bornological;

(ii) $E$ is holomorphically infrabarreled;

(iii) $E$ is hypoholomorphic;

(iv) $E$ has property (D);

(v) $\left(\mathscr{H}(U ; F) ; \mathscr{T}_{0}\right)$ is complete for every non-void open subset $U$ of $E$ and every complete Hausdorff locally convex space $F$;

(vi) $\left(\mathscr{H}(U ; F) ; \mathscr{T}_{0}\right)$ is complete for every equilibrated open subset $U$ of $E$ and every complete Hausdorff locally convex space $F$;

(vii) $E$ is holomorphically Mackey and $\left(\mathscr{H}(U) ; \mathscr{T}_{0}\right)$ is complete for every equilibrated open subset $U$ of $E$;

If, in addition, every compact set in $E$ is sequentially compact; the above conditions are equivalent to the following:

(viii) $E$ is holomorphically semibornological.

Proof. Apply Proposition 1.24 and Proposition 2.5.

ExAmple 1. Let $E$ be a metrizable distinguished locally convex space. The strong dual $E_{b}^{\prime}$ of $E$ is barreled, bornological and $(D F)$, hence Proposition 1.17 implies that $E_{b}^{\prime}$ is polynomially bornological and strongly polynomially barreled. Hence, for $E_{b}^{\prime}$, the conditions (i) - (vii) of Theorem 2.6 are equivalent.

ExAmple 2. A particular case of Example 1 are the $(\mathscr{D} \mathscr{F} \mathscr{M})$-spaces studied in [D] (a given $E$ is said to be a $(\mathscr{D} \mathscr{F} \mathscr{M}$ )-space if $E$ is the strong dual of a Fréchet-Montel space) since every Montel space is distinguished. It follows that a $(\mathscr{D} \mathscr{F} \mathscr{M})$-space is polynomially bornological and strongly

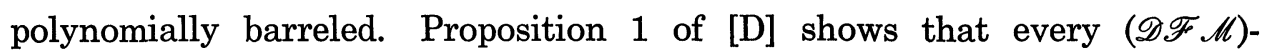
space is a $k$-space, hence hypoholomorphic and, therefore, for such spaces the equivalent conditions of Theorem 2.6 hold. 


\section{§3. The Montel properties}

The Montel properties were introduced by Matos [M] (see also [BMN]). In this section we show that for spaces with polynomially significant properties there are several equivalent conditions involving Montel properties, properties $(D)$ and $\left(D^{*}\right)$ and holomorphically significant properties.

Definition 3.1. A given $E$ has the Montel property (resp. infra-Montel property) if, for every $U$ and every $F$, we have that each collection $\mathscr{X} \subset$ $H(U ; F)$ is relatively compact for $\mathscr{T}_{0}$ if and only if $\mathscr{X}$ is bounded on every finite dimensional compact (resp. compact) subset of $U$, and $\mathscr{X}(x) \subset F$ is relatively compact for every $x \in U$.

In the sequel, the expression " $E$ has property $(M)$ " (resp. " $E$ has property (iM)") signifies that $E$ has the Montel (resp. infra-Montel) property.

Proposition 3.2. If a locally convex space $E$ has property (iM) then $E$ has property $(D)$.

Proof. We follow the proof and notation of Proposition 1.10, (i) $\Rightarrow$ (ii) until we prove that $\mathscr{X}=\left\{f_{m} \mid m \in N\right\}$ is bounded in $\left(\mathscr{H}(V ; F) ; \mathscr{T}_{0}\right)$, and hence $\mathscr{X}$ is bounded in $\left(H(V ; F) ; \mathscr{T}_{0}\right)$. Since $\lim _{m \rightarrow \infty} f_{m}(x)=f(x)$ for all $x$ $\in V$, it follows that $\mathscr{X}(x)$ is relatively compact in $F$ for all $x \in V$. Since $E$ has property $(i M)$, we have that $\mathscr{X}$ is relatively compact in $H(V ; F)$ for $\mathscr{T}_{0}$, which implies that $\mathscr{X}$ is relatively compact in $\mathscr{C}(V ; F)$ for $\mathscr{T}_{0}$. From this point, the proof continues as in Proposition 1.10, (i) $\Rightarrow$ (ii).

Corollary 3.3. For a polynomially infrabarreled space $E$ (see Definition 1.8) the following conditions are equivalent:

(i) $E$ is holomorphically infrabarreled;

(ii) E has property (iM);

(iii) $E$ has property $(D)$.

Proof. The implication (i) $\Rightarrow$ (ii) is known (see [BMN], Proposition 63); that (ii) $\Rightarrow$ (iii) is clear from Proposition 3.2 and that (iii) $\Rightarrow$ (i) follows from Proposition 1.10 .

Corollary 3.4. For a polynomially bornological space $E$ (see Definition 1.5) the following conditions are equivalent:

(i) $E$ is holomorphically bornological;

(ii) $E$ has property (iM);

(iii) $E$ has property $(D)$. 
Proof. The implication (i) $\Rightarrow$ (ii) is clear, that (ii) $\Rightarrow$ (iii) follows from Proposition 3.2 and (iii) $\Rightarrow$ (i) follows from Proposition 1.6.

Proposition 3.5. If a locally convex space $E$ has property $(M)$ then $E$ has property $\left(D^{*}\right)$ (see Definition 1.13).

Proof. The argument is similar to the proof of Proposition 3.2.

Corollary 3.6. For a polynomially barreled space $E$ (see Definition 1.12) the following conditions are equivalent:

(i) $E$ is holomorphically barreled;

(ii) $E$ has property $(M)$;

(iii) $E$ has property $\left(D^{*}\right)$.

Proof. The implication (i) $\Rightarrow$ (ii) is known (see [BMN], Proposition 58), that (ii) $\Rightarrow$ (iii) is clear by Proposition 3.5 and (iii) $\Rightarrow$ (i) follows from Proposition 1.15 .

\section{§4. Holomorphically significant properties of Cauchy regular inductive limits}

In this section we introduce the Cauchy regular inductive limits which are a particular kind of locally convex spaces that embodies an extensive class of spaces of germs as is showed by several examples. We prove that every Cauchy regular inductive limit is polynomially bornological and strongly polynomially barreled. The main result of this section (Theorem 4.6) establishes that in a Cauchy regular inductive limit $E$, properties "holomorphically bornological", "holomorphically barreled", "holomorphically infrabarreled", "Montel", "infra-Montel", $(D),\left(D^{*}\right)$, "completeness of $\left(\mathscr{H}(U ; F) ; \mathscr{T}_{0}\right)$ for all non-void open subsets $U$ of $E$ and all complete Hausdorff locally convex spaces $F$ ' and other properties are equivalent.

Let $\left(E_{m}\right)_{m \in N}$ be an increasing sequence of complex Banach spaces. Denote by $E$ the vector space union of the sequence $\left(E_{m}\right)$ endowed with the inductive topology for the inclusions $E_{m} \subset E$. Given $m \in N$ and $X \subset$ $E_{m}$ let $\mathscr{F}$ be a filter on $X$. The filter $\mathscr{F}$ is said to be $E_{m}$-Cauchy (resp. $E_{m}$-convergent) if $\mathscr{F}$ is a Cauchy filter (resp. convergent filter) for the uniform structure induced on $X$ by $E_{m}$. The filter $\mathscr{F}$ is said to be $E$ Cauchy (resp. $E$-convergent) if $\mathscr{F}$ is a Cauchy filter (resp. convergent filter) for the uniform structure induced on $X$ by $E$.

The inductive limit $E=\underline{\lim _{\longrightarrow}} E_{m}$ is said to be a Cauchy regular inductive 
limit (see [Mu], Definition 1.5) if, for every bounded subset $B$ of $E$, there exists $m \in N$ such that $B$ is contained and bounded in $E_{m}$, and, furthermore, every filter on $B$ is $E$-Cauchy if and only if it is $E_{m}$-Cauchy.

Notation. In the sequel, unless otherwise stated, the notation

$$
E=\underset{\lim }{\longrightarrow} E_{m}
$$

denotes a Cauchy regular inductive limit where $\left(E_{m}\right)$ is an increasing sequence of (complex) Banach spaces and $E$ is the union of the sequence $\left(E_{m}\right)$. We suppose also that $E$, endowed with the inductive topology for the inclusions $E_{m} \subset E$, is a Hausdorff locally convex space. For all $m \in N$, the continuous inclusions $E_{m} \subset E_{m+1}$ and $E_{m} \subset E$ are denoted by $j_{m}$ and $i_{m}$ respectively and we suppose that $\left\|j_{m}\right\|=1$.

ExAmple 1. Every complex Banach space is clearly a Cauchy regular inductive limit.

ExAmple 2. If $X$ and $Y$ are complex Banach spaces and $K$ is a compact subset of $X$, then the space

$$
\left(\mathscr{H}(K ; Y) ; \mathscr{T}_{\omega}\right)
$$

of holomorphic germs on $K$ with values in $Y$ endowed with the Nachbin topology $\mathscr{T}_{\omega}$, is a Cauchy regular inductive limit (see [Ch], Proposition 3.2 and Proposition 3.8).

ExAmple 3. If $X$ is a metrizable and quasi-normable locally convex complex space and $K$ is a compact subset of $X$, then the space

$$
\left(\mathscr{H}(K) ; \mathscr{T}_{\omega}\right)
$$

of holomorphic germs on $K$ with complex values, endowed with the Nachbin topology $\mathscr{T}_{\omega}$, is a Cauchy regular inductive limit (see [A-Mu], Theorem 2).

EXAMPle 4. Let $\left(X_{m}\right)_{m \in N}$ be an increasing sequence of (complex) Banach spaces, $X=\cup_{m \in N} X_{m}$ and suppose that $X=\lim X_{m}$ is a strict inductive limit (i.e. $X_{m}$ has the induced topology of $X_{m+1}$, for all $m \in N$ ). Then from Dieudonné-Schwartz theorem (see $[\mathrm{H}], \mathrm{Ch} .2, \S 12$ ) it follows that $X$ is a Cauchy regular inductive limit.

LEMMA 4.1. Let $B$ be a bounded set in a Cauchy regular inductive limit $E=\underset{\lim }{\longrightarrow} E_{m}$. Then, there exists $m \in N$ such that $B$ is contained and bounded 
in $E_{m}$ and, furthermore, $E$ and $E_{m}$ induce the same topology on $B$, hence $B$ is metrizable. In particular, every compact subset of $E$ is sequentially compact.

Proof. Since $E$ is a Cauchy regular inductive limit, there exists $m \in$ $N$ such that $B$ is contained and bounded in $E_{m}$, and furthermore, the condition on filters on $B$ holds. Let $\mathscr{T}$ (resp. $\mathscr{T}_{m}$ ) be the topology induced on $B$ by $E$ (resp. $E_{m}$ ); clearly $\mathscr{T} \leq \mathscr{T}_{m}$. Suppose $B$ closed in $E_{m}$, fix any $x \in B$ and denote by $\mathscr{V}(x)$ (resp. $\mathscr{V}_{m}(x)$ ) the filter of $\mathscr{T}$-neighborhoods (resp. $\mathscr{T}_{m}$-neighborhoods) of $x$. Since $B$ is closed, the condition on filters on $B$ implies that $\mathscr{V}(x) \supset \mathscr{V}_{m}(x)$, hence $\mathscr{T} \geq \mathscr{T}_{m}$. The case of an arbitrary $B$ follows from this. Then:

Proposition 4.2. Let $E=\underline{\lim _{m}} E_{m}$ be a Cauchy regular inductive limit.

(a) $E$ is barreled, bornological, $(D F)$ and quasi-normable.

(b) If $K$ is a compact subset of $E$, there exists $m \in N$ such that $E$ and $E_{m}$ induce the same topology on $K$ and hence $K$ is metrizable.

(c) $E$ is complete.

Proof. (a) Since $E$ is an inductive limit of Banach spaces, it follows that $E$ is barreled, bornological and $(D F)$. Hence $E$ is quasi-normable since $E$ is infrabarreled.

(b) Follows from Lemma 4.1.

(c) Since $E$ is a $(D F)$-space it is enough to show that $E$ is quasicomplete. Let $\mathscr{F}$ be a Cauchy filter on a bounded closed subset $X$ of $E$. Since $E$ is a Cauchy regular inductive limit, there exist $m \in N$ such that $X$ is bounded and closed in $E_{m}$, and furthermore, every $E$-Cauchy filter on $X$ is a $E_{m}$-Cauchy filter. It follows that $\mathscr{F}$ is a $E_{m}$-Cauchy filter, hence $\mathscr{F}$ is $E_{m}$-convergent, whence $\mathscr{F}$ is $E$-convergent.

Remark. The inductive limit $E=\underset{\lim }{\longrightarrow} E_{m}$ is said to be a compactly regular inductive limit if, for every compact subset $K$ of $E$, there exist $m \in N$ such that $K$ is contained and compact in $E_{m}$. It is known that $E$ is a compactly regular inductive limit if and only if $E$ is a Cauchy regular inductive limit. Then:

THEOREM 4.3. Let $E=\underline{\lim _{\longrightarrow}} E_{m}$ be a Cauchy regular inductive limit. (a) $E$ is polynomially bornological (see Definition 1.5). 
(b) $E$ is strongly polynomially barreled and hence polynomially barreled (see Definition 1.12 and Definition 1.16).

(c) $E$ is locally holomorphically infrabarreled (see Definition 1.18).

Proof. The statements (a) and (b) follows from Proposition 4.2 (a) and Proposition 1.17. We shall prove the statement (c). Given $U$ and $F$, let $\mathscr{X}$ be a $\mathscr{T}_{0}$-bounded subset of $\mathscr{H}(U ; F)$. If $K$ is a compact subset of $U$, there exists $m \in N$ such that $K$ is contained and compact in $U_{m}=U \cap E_{m}$. Since the set $\mathscr{X}_{m}=\left\{f\left|U_{m}\right| f \in \mathscr{X}\right\}$ is a $\mathscr{T}_{0}$-bounded subset of $\mathscr{H}\left(U_{m} ; F\right)$ and $E_{m}$ is locally holomorphically infrabarrelled, it follows that $\mathscr{X}_{m}|K=\mathscr{X}| K$ is equicontinuous.

Lemma 4.4. If $E=\lim _{\longrightarrow} E_{m}$ is a Cauchy regular inductive limit, then for every $U$ and every $F, \overrightarrow{w e}$ have (see Definition 1.1 and Definition 1.13)

$$
\mathscr{H}_{D^{*}}(U ; F)=\mathscr{H}_{D}(U ; F) .
$$

Proof. Let $f \in \mathscr{H}_{D^{*}}(U ; F)$ and let $K$ be a compact subset of $U$. There exists $m \in N$ such that $K$ is contained and compact in $U_{m}=U \cap E_{m}$. Let $f_{m}=f \mid U_{m}$; then the relations

$$
\hat{\partial}^{n} f_{m}(\xi)=\hat{\partial}^{n} f(\xi) \circ i_{m}, \quad \text { for all } n \in N \text { and all } \xi \in U_{m}
$$

imply that $f_{m} \in \mathscr{H}_{D^{*}}\left(U_{m} ; F\right)$. Since $E_{m}$ is holomorphically barreled, by Proposition 1.15 it follows that $f_{m} \in \mathscr{H}\left(U_{m} ; F\right)$, hence $f(K)=f_{m}(K)$ is bounded in $F$.

Lemma 4.5. For a Cauchy regular inductive limit $E$ the following conditions are equivalent:

(i) $E$ is holomorphically bornological;

(ii) $E$ is holomorphically barreled;

(iii) $E$ has property $\left(D^{*}\right)$.

Proof. (i) $\Rightarrow$ (ii): By Proposition 1.6, $E$ has property $(D)$, hence by Lemma $4.4 E$ has property $\left(D^{*}\right)$. By Theorem 4.3 (b), $E$ is polynomially barreled, hence (ii) follows from Proposition 1.15. For (ii) $\Rightarrow$ (iii) apply Proposition 1.15. That (iii) $\Rightarrow$ (i) is clear since $\left(D^{*}\right) \Rightarrow(D)$ and $E$ is polynomially bornological by Theorem 4.3 (a).

Now we can prove the main result of this paper.

TheOREM 4.6. For a Cauchy regular inductive limit $E$ the following conditions are equivalent: 
(i ) E is holomorphically bornological;

(ii) $E$ is holomorphically barreled;

(iii) $E$ is holomorphically infrabarreled;

(iv) $E$ has property $(D)$;

( v ) $E$ has property $\left(D^{*}\right)$;

(vi ) $E$ is holomorphically semibornological (see Definition 1.21);

(vii) $E$ is hypoholomorphic;

(viii) $\left(\mathscr{H}(U ; F) ; \mathscr{T}_{0}\right)$ is complete for every non-void open subset $U$ of $E$ and every complete Hausdorff locally convex space $F$;

(ix) $\left(\mathscr{H}(U ; F) ; \mathscr{T}_{0}\right)$ is complete for every equilibrated open subset $U$ of $E$ and every complete Hausdorff locally convex space $F$;

( x ) $E$ is holomorphically Mackey and $\left(\mathscr{H}(U) ; \mathscr{T}_{0}\right)$ is complete for every equilibrated open subset $U$ of $E$;

(xi) $E$ has property (M) (see Definition 3.1);

(xii) $E$ has property (iM) (see Definition 3.1).

Proof. Since by Theorem 4.3 (a), $E$ is polynomially bornological and every compact subset of $E$ is sequentially compact (by Proposition 4.2 (b)) the equivalences

$$
\text { (i) } \Longleftrightarrow \text { (iii) } \Longleftrightarrow \text { (iv) } \Longleftrightarrow \text { (vi) } \Longleftrightarrow \text { (vii) } \Longleftrightarrow \text { (viii) } \Longleftrightarrow \text { (ix) } \Longleftrightarrow \text { (x) }
$$

follow from Theorem 2.6. By Lemma 4.5 we have (i) $\Leftrightarrow$ (ii) $\Leftrightarrow$ (v). Finally Theorem 4.3 (a) (resp. (b)) and Corollary 3.4 (resp. Corollary 3.6) imply that (i) $\Leftrightarrow$ (xii) (resp. (ii) $\Leftrightarrow$ (xi)).

Proposition 4.7. For a Cauchy regular inductive limit $E=\underline{\lim } E_{m}$, the following conditions are equivalent:

(i) $E$ is a Silva space;

(ii) $E$ is a Schwartz space;

(iii) $E$ is a Montel space.

Proof. (i) $\Rightarrow$ (ii): Since $E$ is Hausdorff and quasi-normable, it is enough to show that every bounded subset of $E$ is precompact. Given a bounded subset $L$ of $E$, there exists $m \in N$ such that $L$ is contained and bounded in $E_{m}$, hence, if we denote by $B$ the unit ball of $E_{m}$, there exists $r>0$ such that $r L \subset B$. From (i) it follows that $j_{m}(r L)=r j_{m}(L)=r L$ is relatively compact in $E_{m+1}$, whence $L$ is a precompact subset of $E$.

(ii) $\Rightarrow$ (iii): Let $L$ be a bounded subset of $E$. By (ii), $L$ is a precom- 
pact subset of $E$, hence $L$ is a relatively compact subset of $E$, since $E$ is complete by Proposition 4.2, (c).

(iii) $\Rightarrow(\mathrm{i})$ : It is enough to show that there exists a subsequence $\left(E_{m_{i}}\right)_{i \in N}$ of $\left(E_{m}\right)_{m \in N}$ such that the inclusions $h_{i}: E_{m_{i}} \rightarrow E_{m_{i+1}}$ are compact for all $i \in N$. We take $m_{o}=0$ and suppose $m_{1}, \cdots, m_{s}$ are defined such that $m_{o}<m_{1}<\cdots<m_{s}$ and such that the inclusions $h_{i}$ are compact for $i=0,1, \cdots, s-1$. Let $B$ be the unit ball in $E_{m_{s}}$, then the set $i_{m_{s}}(B)=B$ is bounded in $E$, hence by (iii), $B$ is relatively compact in $E$. It follows that there exists $p \in N, p>m_{s}$ such that $B$ is contained and relatively compact in $E_{p}$. We define then $m_{s+1}=p$.

EXAmple 5. An infinite dimensional complex Banach space is a trivial example of a Cauchy regular inductive limit which satisfies the equivalent conditions of Theorem 4.6 but which is not a Silva space.

EXAMPLE 6. Let $X$ be a metrizable Schwartz locally convex complex space and let $K$ be a compact subset of $X$. Then $\left(\mathscr{H}(K) ; \mathscr{T}_{\omega}\right)$ is a Silva space (see [Bi-Me] and [A-Mu]). Since $X$ is in particular quasi-normable, $\left(\mathscr{H}(K) ; \mathscr{T}_{\omega}\right)$ is a Cauchy regular inductive limit, hence this space is an example of a Cauchy regular inductive limit which satisfies the equivalent conditions of Theorem 4.6.

EXAMPLE 7. We shall show that there exist Cauchy regular inductive limits which are not holomorphically bornological. For this we consider the following example (see [BMN], Example 18). Let $X_{0}$ be an infinite dimensional complex Banach space and let $\left(X_{m}\right)_{m \geq 1}$ be the sequence defined by $X_{m}=C$ for all $m \geq 1$. We set

$$
E=\bigoplus_{m=0}^{\infty} X_{m} \quad \text { and } \quad E_{m}=\bigoplus_{i=0}^{m} X_{i}, \quad \text { for all } m \in N .
$$

We consider every $E_{m}$ equipped with its natural Banach structure and we endow $E$ with the locally convex direct sum topology. It is easy to see that this topology on $E$ coincides with the inductive topology for the sequence of inclusions

$$
i_{m}:\left(x_{0}, \cdots, x_{m}\right) \in E_{m} \longmapsto\left(x_{0}, \cdots, x_{m}, 0,0, \cdots\right) \in E .
$$

By using well-known properties of locally convex direct sums, it follows that $E=\underset{\lim }{\longrightarrow} E_{m}$ is a Cauchy regular inductive limit. On the other hand, this space is not holomorphically bornological, as is proved in [BMN], Example 18. 
We shall end this section with a characterization of Cauchy regular inductive limits which are not metrizable.

Definition 4.8. We say that a Cauchy regular inductive limit $E=$ $\underset{\longrightarrow}{\longrightarrow} E_{m}$ is proper if $E_{m} \neq E$ for all $m \in N$.

Clearly a proper Cauchy regular inductive limit is not a normed space. Indeed, assume that $E$ is normed, then by Proposition 4.2 (c), $E$ is actually a Banach space. Since $E$ is a proper Cauchy regular inductive limit it follows that, for all $m \in N$, the inclusion $i_{m}: E_{m} \rightarrow E$ is not surjective. Hence, by the open mapping theorem, $E_{m}$ is meager in $E$ for all $m \in N$ and since $E$ is the union of the sequence $\left(E_{m}\right)$ it follows that $E$ is the union of a sequence of rare sets. This is a contradiction.

Proposition 4.9. For a Cauchy regular inductive limit $E=\underset{\lim }{\rightarrow} E_{m}$ the following conditions are equivalent:

(i) $E_{m} \neq E$ for all $m \in N$ (i.e. $E$ is proper);

(ii) There exists a subsequence $\left(E_{m_{i}}\right)$ of $\left(E_{m}\right)$ such that $E_{m_{i}} \neq E_{m_{i+1}}$ for all $i \in N$;

(iii) $E$ is not metrizable.

Proof. The implications (i) $\Rightarrow$ (ii) and (iii) $\Rightarrow$ (i) are trivial. For (ii) $\Rightarrow$ (iii) apply de Mackey countability condition.

\section{§5. Miscellaneous results}

Our first objective in this section is to find a sufficient condition on a Cauchy regular inductive limit so that it is not holomorphically bornological. We shall need the following lemma.

Lemma 5.1. Let $U$ be a non-void open subset of a locally convex space $E$, let $F$ be a locally convex space, let $f \in \mathscr{H}_{D}(U ; F)$ and $\xi \in U$. Then, the following conditions are equivalent:

(i) $f$ is continuous at $\xi$;

(ii) $f$ is amply bounded at $\xi$;

(iii) For every $\beta \in C S(F)$, there exists $\alpha \in C S(E), \alpha \neq 0$ such that,

a) $1 / m ! \hat{\partial}^{m} f(\xi) \in \mathscr{P}\left({ }^{m} E_{\alpha} ; F_{\beta}\right)$, for all $m \in N$.

b) $\sup _{m \in N}\left\|1 / m ! \hat{\partial}^{m} f(\xi)\right\|_{\alpha, \beta}<\infty$.

Proof. That (i) $\Rightarrow$ (ii) is clear. We shall prove that (ii) $\Rightarrow$ (iii). Assume first that $F$ is Hausdorff. Given $\beta \in C S(F)$, since $f$ is amply bounded at $\xi$, there exists $\alpha \in C S(E), \alpha \neq 0$ such that 


$$
\bar{B}_{\alpha, 1}(\xi) \subset U \text { and } S=\sup \{\beta(f(x)) \mid \alpha(x-\xi) \leq 1\}<\infty .
$$

Since $\xi+\lambda t \in \bar{B}_{\alpha, 1}(\xi)$ for all $\lambda \in C,|\lambda| \leq 1$ and all $t \in \bar{B}_{\alpha, 1}(0)$, by the Cauchy integral formula we have

$$
\left\|\frac{1}{m !} \hat{\partial}^{m} f(\xi)\right\|_{\alpha, \beta}<S \quad \text { for all } m \in N,
$$

which proves (a) and (b) in this case. Assume now that $F$ is arbitrary. Let $\tilde{F}$ be the Hausdorff space associated which $F$ and let $q: F \rightarrow \tilde{F}$ be the canonical map, then we have

$$
\tilde{f}=q \circ f \in \mathscr{H}_{D}(U ; \tilde{F}) .
$$

Given $\beta \in C S(F)$, let $\bar{\beta} \in C S(\tilde{F})$ be the seminorm defined by $\bar{\beta} \circ q=\beta$, then by the preceding case, associated to $\bar{\beta}$ and $\tilde{f}$, we can find $\alpha \in C S(E)$ such that

$$
\frac{1}{m !} \hat{\partial}^{m} \tilde{f}(\xi) \in \mathscr{P}\left({ }^{m} E_{\alpha} ; \tilde{F}_{\beta}\right), \quad \text { for all } m \in N
$$

and

$$
\sup _{m \in N}\left\|\frac{1}{m !} \hat{\partial}^{m} \tilde{f}(\xi)\right\|_{\alpha, \tilde{\beta}}<\infty
$$

which implies the statements (a) and (b).

(iii) $\Rightarrow$ (i): Fix any $\beta \in C S(F)$. We denote by $r: F_{\beta} \rightarrow F / \beta$ the canonical map and we denote by $\dot{\beta}$ the norm induced by $\beta$ on $F / \beta$. Let $g=r \circ f$. By (iii) there exists $\alpha \in C S(E)$ such that

$$
\frac{1}{m !} \hat{\partial}^{m} g(\xi) \in \mathscr{P}\left({ }^{m} E_{\alpha} ; F / \beta\right) \quad \text { for all } m \in N
$$

and

$$
S=\sup _{m \in N}\left\|\frac{1}{m !} \hat{\partial}^{m} g(\xi)\right\|_{\alpha, \dot{\beta}}<\infty
$$

Since $g \in \mathscr{H}_{D}(U ; F / \beta)$, if $V$ is a $\xi$-equilibrated neighborhood of $\xi$ in $U$, we have

$$
g(x)=\sum_{m=0}^{\infty} \frac{1}{m !} \hat{\partial}^{m} g(\xi)(x-\xi), \quad \text { pointwise in } V
$$

Given $\varepsilon>0$, let $r$ be a real number such that $0<r<1$ and $\operatorname{Sr}(1-r)^{-1}$ $\leq \varepsilon$, then for all $x \in B_{\alpha, r}(\xi) \cap V$, we get 


$$
\begin{aligned}
\beta(f(x)-f(\xi)) & =\dot{\beta}(g(x)-g(\xi)) \leq \sum_{m=1}^{\infty}\left\|\frac{1}{m !} \hat{\partial}^{m} g(\xi)\right\|_{\alpha, \dot{\beta}} \alpha(x-\xi)^{m} \\
& \leq S r(1-r)^{-1} \leq \varepsilon .
\end{aligned}
$$

Proposition 5.2. Let $E=\underset{\lim }{\longrightarrow} E_{\nu}$ be a proper Cauchy regular inductive limit and denote by $B_{r}^{\nu}$ the open ball of center 0 and radius $r$ in the space $E_{\nu}$ for all $\nu \in N$. Assume that the following conditions is fulfilled:

(*) There exists a normed space $G$, there exists a sequence $\left(P_{m}\right)_{m \in N}$ where $P_{m} \in \mathscr{P}\left({ }^{m} E ; G\right)$ for all $m \in N$ and there exists $r>0$ such that:

(a) The series

$$
\sum_{m=0}^{\infty}\left(P_{m} \mid E_{\nu}\right)(x)
$$

is pointwise convergent for every $x \in B_{r}^{\nu}$ and all $\nu \in N$.

(b) For every $\alpha \in C S(E)$ we have

$$
\sup _{m \in N}\left\|P_{m}\right\|_{\alpha}=\infty \text {. }
$$

Then, $E$ is not holomorphically bornological.

Proof. We shall construct an open subset $W$ of $E$ and a $D$-holomorphic mapping $f$ of $W$ into $G$ such that $f$ is discontinuous. Since $\left\|j_{\nu}\right\|=1$ for all $\nu \in N$ we have $B_{r}^{\nu} \subset B_{r}^{\nu+1}$ for all $\nu \in N$. Hence $V$, the union of the sequence $\left(B_{r}^{\nu}\right)_{\nu \in N}$, is a neighborhood of 0 in $E$, we set $W=\stackrel{\circ}{V}$. Clearly the series

$$
\sum_{m=0}^{\infty} P_{m}(x)
$$

is pointwise convergent for all $x \in V$, hence we can define a mapping

$$
f: x \in W \longmapsto \sum_{m=0}^{\infty} P_{m}(x) \in G .
$$

We shall show that $f$ is algebraically holomorphic. Let $S$ be a finite dimensional linear subspace of $E$, then there exists $k \in N$ such that $S \subset$ $E_{k}$. Fix $\xi \in W \cap S$, it is enough to prove that there exists an open neighborhood $A$ of $\xi$ in $W \cap S$ such that $f \mid A \in \mathscr{H}(A ; G)$. Clearly, there exists $n \geq k$ such that $\xi \in B_{r}^{n}$. Consider, for all $\nu \in N$, the mapping

$$
g_{\nu}: x \in B_{r}^{\nu} \longmapsto \sum_{m=0}^{\infty}\left(P_{m} \mid E_{\nu}\right)(x) \in G
$$


Hypothesis (a) implies $g_{v} \in \mathscr{H}\left(B_{r}^{\nu} ; G\right)$ for all $\nu \in N$. Since $B_{r}^{n} \cap S$ and $W \cap$ $S$ are open sets in $S$ containing $\xi$, the set

$$
A=B_{r}^{n} \cap W \cap S
$$

is an open set in $S$ containing $\xi$ and, since

$$
f\left|A=g_{n}\right| A \in \mathscr{H}(A ; G)
$$

it follows that $f \in \mathscr{H}_{a}(W ; G)$. Next we set $W_{\nu}=W \cap E_{\nu}$ for all $\nu \in N$ and we shall show that

$$
f_{\nu}=f \mid W_{\nu} \in \mathscr{H}\left(W_{\nu} ; G\right), \quad \text { for all } \nu \in N .
$$

Fix any $\nu \in N$. Since $f \in \mathscr{H}_{a}(W ; G)$ we have $f_{\nu} \in \mathscr{H}_{a}(W ; G)$ and, since $W_{\nu}$ is a connected open set in $E_{\nu}$, by a classical theorem of Zorn, it is enough to show that the set $X$ of points of continuity of $f_{\nu}$ in $W_{\nu}$ is non-void. Indeed, this results follow from the two statements:

$$
g_{\nu} \in \mathscr{H}\left(B_{r}^{\nu} ; G\right) \text { and } f\left|W \cap B_{r}^{\nu}=g_{\nu}\right| W \cap B_{r}^{\nu}
$$

which proves that $\emptyset \neq W \cap B_{r}^{\nu} \subset X$. Hence $f \in \mathscr{H}_{c}(W ; G)$. Indeed, if $K$ is a compact subset of $W$, there exists $\nu \in N$ such that $K$ is contained and compact in $W_{\nu}=W \cap E_{\nu}$. Since $f_{\nu}=f \mid W_{\nu} \in \mathscr{H}\left(W_{\nu} ; G\right)$ it follows that $f(K)$ is bounded. We shall show finally that $f$ is discontinuous. By the uniqueness of the Taylor expansion, we have

$$
P_{m}=\frac{1}{m !} \hat{\partial}^{m} f(0), \quad \text { for all } m \in N .
$$

Hence, given $\alpha \in \mathrm{CS}(E), \alpha \neq 0$, hypothesis (b) implies

$$
\sup _{m \in N}\left\|\frac{1}{m !} \hat{\partial}^{m} f(0)\right\|_{\alpha}=\infty
$$

which implies, by Lemma 5.1 , that $f \notin \mathscr{H}(W ; G)$.

In the following result we denote by

$$
\left(\mathscr{P}\left({ }^{m} E\right)\right)_{i}
$$

the space $\mathscr{P}\left({ }^{m} E\right)$ endowed with the inductive topology defined by the identity maps id: $\mathscr{P}\left({ }^{m} E_{\alpha}\right) \rightarrow \mathscr{P}\left({ }^{m} E\right)$. We denote $\mathscr{H}(K)$ (resp. $\mathscr{H}(K ; Y)$ ) the locally convex space $\left(\mathscr{H}(K) ; \mathscr{T}_{\omega}\right)$ (resp. $\left.\left(\mathscr{H}(K ; Y) ; \mathscr{T}_{\omega}\right)\right)$ of holomorphic germs on $K$ with complex values (resp. with values in $Y$ ), endowed with the topology $\mathscr{T}_{\omega}$. 
Proposition 5.3. Let $E$ be a locally convex space, let $K$ be a compact subset of $E$ and assume that $\mathscr{H}(K)$ is holomorphically bornological. Then: (a) $\mathscr{P}\left(\left({ }^{m} E\right)\right)_{i}$ is holomorphically bornological for all $m \in N$.

(b) If $E$ is metrizable and distinguished, then the strong dual $E_{b}^{\prime}$ of $E$ is holomorphically bornological.

Proof. (a) The space $\left(\mathscr{P}\left({ }^{m} E\right)\right)_{i}$ is a quotient of $\mathscr{H}(K)$ (see [A-Mu], Proposition 1), hence this statement follows from the fact that every quotient of a holomorphically bornological space is holomorphically bornological.

(b) The assumption on $E$ implies that $E_{b}^{\prime}$ is isomorphic to $E_{i}^{\prime}=$ $\left(\mathscr{P}\left({ }^{1} E\right)\right)_{i}$ (see [A-Mu], Corollary of Proposition 1), hence the result follows from (a).

Proposition 5.4. Let $X$ be a complex normed space, let $Y$ be a complex Banach space and let $K$ be a compact subset of $X$. Then, the following conditions are equivalent:

(i) $\mathscr{H}(K ; Y)$ is a Silva space;

(ii) $X$ and $Y$ are finite dimensional spaces.

Proof. That (ii) $\Rightarrow$ (i) is well-known, so it remains to show that (i) $\Rightarrow$ (ii). The hypothesis implies that $\mathscr{H}(K ; Y)$ is a semi-Montel space, hence the Banach space $\mathscr{L}(X ; Y)$ is also a semi-Montel space since $\mathscr{L}(X ; Y)$ is a closed subspace of $\mathscr{H}(K ; Y)$ (see [Ch], Proposition 7.2).

If $U$ is a non-void open subset of a complex locally convex space $E$ and $F$ is a complex Banach space, we denote by

$$
\mathscr{H}^{\infty}(U ; F)
$$

the complex Banach space of all bounded holomorphic mappings of $U$ into F.

LEMMA 5.5. Let $E$ be a complex metrizable locally convex space and let $F$ be an infinite dimensional complex Banach space. Assume that $U$ and $V$ are non-void open subsets of $E$ such that $V \subset U$. Then the restriction map

$$
j: f \in \mathscr{H}^{\infty}(U ; F) \longmapsto f \mid V \in \mathscr{H}^{\infty}(V ; F)
$$

is not compact.

Proof. Let $B$ be the open unit ball of $\mathscr{H}^{\infty}(U ; F)$ and assume that $j$ is compact. Fix any $\xi \in V$, then it is clear that the mapping 


$$
u: f \in \mathscr{H}^{\infty}(V ; F) \longmapsto f(\xi) \in F
$$

is linear and continuous. Since $j$ is compact, $j(B)$ is relatively compact in $\mathscr{H}^{\infty}(V ; F)$, hence the set

$$
u(j(B))=\{f(\xi) \mid f \in B\}=B(\xi)
$$

is relatively compact in $F$. On the other hand, the composed map

$$
v=u \circ j: f \in \mathscr{H}^{\infty}(U ; F) \longmapsto f(\xi) \in F
$$

is linear, continuous and surjective and $v(B)=B(\xi)$. By the open mapping theorem, it follows that $v$ is open, hence $B(\xi)$ is open in $F$. This is a contradiction, since $F$ is infinite dimensional.

Proposition 5.6. Let $K$ be a compact subset of a metrizable complex locally convex space $E$ and let $F$ be an infinite dimensional complex Banach space. Then $\mathscr{H}(K ; F)$ is not a Silva space.

Proof. Since $E$ is metrizable, $K$ has a decreasing countable basis of open neighborhoods $\left(U_{m}\right)_{m \in N}$ and

$$
\mathscr{H}(K ; F)=\lim _{\longrightarrow} \mathscr{H}^{\infty}\left(U_{m} ; F\right) .
$$

Assume that $\mathscr{H}(K ; F)$ is a Silva space. Then, there exists an increasing sequence $\left(B_{p}\right)_{p \in N}$ of complex Banach spaces such that the following statements are fulfilled:

(I) $\mathscr{H}(K ; F)$ is the union of the sequence $\left(B_{p}\right)$.

(II) The topology $\mathscr{T}_{\omega}$ on $\mathscr{H}(K ; F)$ is the inductive topology defined by the inclusions $B_{p} \longrightarrow \mathscr{H}(K ; F)(p \in N)$.

(III) For all $p \in N$, the inclusion $i_{p}: B_{p} \longleftrightarrow B_{p+1}$ is compact.

By a well-known result of Grothendieck (see [Mu], Proposition 1.4) for every $m \in N$ there exist $p=p_{m} \in N$ and a continuous linear mapping $u_{p}$ such that the following diagram

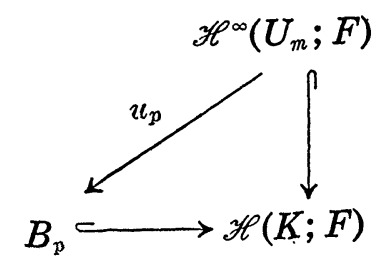

commutes. The same argument applied to $B_{p+1}$ shows that there exists $n \in N$ and a continuous linear mapping $v$ such that the following diagram 


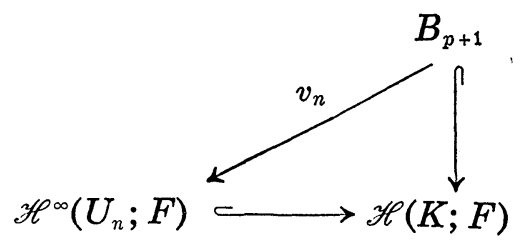

commutes. Since the sequence $\left(\mathscr{H}^{\infty}\left(U_{\nu} ; F\right)\right)_{\nu \in N}$ is increasing, we may assume that $n \geq m$. Hence we have a restriction map

$$
j: \mathscr{H}^{\infty}\left(U_{m} ; F\right) \longrightarrow \mathscr{H}^{\infty}\left(U_{n} ; F\right)
$$

and a commutative diagram of linear continuous mappings:

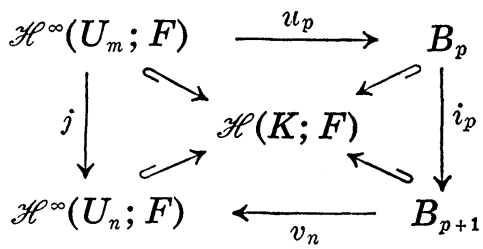

Hence, we have $j=v_{n} \circ i_{p} \circ u_{p}$ and, since $i_{p}$ is compact, it follows that $j$ is compact. This is a contradiction, in view of Lemma 5.5.

\section{REFERENCES}

[A-Mu] P. Aviles and J. Mujica, Holomorphic germs and homogeneous polynomials on quasi-normale metrizable spaces, Rendiconti di Matematica, to appear.

[B] J. A. Barroso, Topologias nos espaços de aplicações holomorfas entre espaços localmente convexos, An. Acad. Bras. de Ciências, 43, no. 3/4 (1971), 527-546.

[BMN] J. A. Barroso, M. A. Matos and L. Nachbin, On holomorphy versus linearity in classifying locally convex spaces, Infinite Dimensional Holomorphy and Applications (Editor: M. C. Matos), North-Holland Mathematics Studies (1977).

[Bi-Me] K. D. Bierstedt and R. Meise, $\mathscr{H}(K)$ et $\left(\mathscr{H}(U) ; \mathscr{T}_{\omega}\right)$ sur des espaces metrisables nucléaires ou de Schwartz, C. R. Acad. Sc. Paris, t. 283 (1976).

[Ch] S. B. Chae, Holomorphic germs on Banach spaces, Ann. Inst. Fourier, 21, 3 (1971), 107-141.

[D] S. Dineen, Holomorphic functions on strong duals of Fréchet-Montel spaces, Infinite Dimensional Holomorphy and Applications (Editor: M. C. Matos), North-Holland Mathematics Studies (1977).

[G] A. Grothendieck, Espaces vectoriels topologiques, Publicação da Sociedade Matemática de São Paulo, $3^{\circ}$ Edição (1964).

[H] J. Horvath, Topological vector spaces and distributions, Vol. I, Addison Wesley (1966).

[M] M. C. Matos, On locally convex spaces with the Montel property, Functional Analysis (Editor: D. G. de Figueiredo), Marcel Dekker (1976), 245-250.

[Mu] J. Mujica, Spaces of germs of holomorphic functions, Advances in Mathematics, 4 (1979). 
[N1] L. Nachbin, A glimpse at infinite dimensional holomorphy, Proceedings on Infinite Dimensional Holomorphy (Editors: T. L. Hayden; T. J. Suffridge), Lecture Notes in Mathematics, Springer-Verlag, 364 (1974), 69-78.

[N2] - Some holomorphically significant properties of locally convex spaces, Functional Analysis (Editor: D. G. de Figueiredo), Marcel Dekker (1976), 251-277.

[S] L. R. Soraggi, Partes limitadas nos espaços de germes de aplicações holomorfas, Thesis, Universidade Federal do Rio de Janeiro, no. 4 (1976).

[W] A. J. M. Wanderley, Germes de aplicações holomorfas em espaços localmente convexos, Thesis, Universidade Federal do Rio de Janeiro.

Universidade de São Paulo

Instituto de Matemática e Estatistica

São Paulo

Brasil 